\title{
Local Subcutaneous Injection of Erythropoietin Might Improve Fat Graft Survival, Whereas Continuous Infusion Using an Osmotic Pump Device Was Harmful by Provoking an Overwhelming Foreign Body Reaction in a Nude Mouse Model
}

Eun Key Kim, Yeonhoon Lee, Hee Jong Lee, Joon Pio Hong

Department of Plastic and Reconstructive Surgery, Asan Medical Center, University of Ulsan College of Medicine, Seoul, Korea
No potential conflict of interest relevant to this article was reported.
Background Since the survival of grafted fat requires successful revascularization, we hypothesized that local delivery of erythropoietin (EPO), a hemangiogenic and antiapoptotic factor, might enhance the survival of fat grafts in a nude mouse model. Methods Our experiment consisted of 2 parts. The first involved direct injection of EPO or saline at the grafting site before fat grafting and for 4 days afterwards. In the second part, EPO or saline was delivered continuously for 1 week via an osmotic pump device, and a group without an implantable pump represented the control group. After 16 weeks, the surviving fat grafts were weighed and subjected to histomorphometry. Results In the first experiment, fat graft survival was higher in the EPO-injected group, but not statistically significant ( $69.2 \%$ vs. $59.2 \% ; \mathrm{P}=0.21$ ). Histomorphometry showed significantly higher levels of microvessel formation and less cystic degeneration in the EPO-injected group. In the second experiment, the survival rate was significantly lower in both pump groups than in the control group (40.7\% in the EPO pump group, $7.7 \%$ in the saline pump group, and $66.7 \%$ in the control group).

Conclusions Local injection of EPO into the fat graft site appeared to improve graft survival, with increased angiogenesis, suppressed inflammation, and decreased degeneration. However, the insertion of an osmotic pump exerted detrimental effects on graft survival and the histomorphometric profiles of the fat graft compared with the control group due to the overwhelming foreign body reaction.

Keywords Adipose tissue, Erythropoietin, Graft survival, Inflammation
Received: May 17, 2018 Revised: Jul 11, 2018 Accepted: Jul 11, 2018 Correspondence: Eun Key Kim Department of Plastic and Reconstructive Surgery, Asan Medical Center, University of Ulsan College of Medicine, 88 Olympic-ro 43-gil, Songpa-gu, Seoul 05505, Korea. E-mail: nicekek@korea.com Copyright @ 2018 The Korean Society for Aesthetic Plastic Surgery.

This is an Open Access article distributed under the terms of the Creative Commons Attribution Non-Commercial License (http://creativecommons.org/licenses/by-nc/4.0/) which permits unrestricted non-commercial use, distribution, and reproduction in any medium, provided the original work is properly cited. www.e-aaps.org

\section{INTRODUCTION}

Free fat injection has become a common practice for various types of soft tissue augmentation, and its new role as a driving force in tissue regeneration has been described in recent publications [1,2]. However, inconsistency in long-term outcomes remains a drawback and there is no universal agreement on the best methodology or the necessary amount of overcorrection when performing a free fat injection [3-5].

The survival of grafted fat requires successful revascularization. A study of mice treated with an angiogenesis inhibitor demonstrat- 
ed that the degree of revascularization determined the volume retention and influenced gene expression [6]. Other studies have attempted, with varying degrees of success, to augment graft survival using various growth factors, cytokines, or platelet-rich plasma (PRP) [7-11]. An increasing number of studies have implanted fat particles mixed with adipose-derived stem cells (ASCs) with promising results $[12,13]$. However, bedside application of this method seems unlikely in the near future due to excessive costs and other clinical safety issues.

Erythropoietin (EPO) is a potent hemangiogenic factor that, with the ubiquitous expression of its receptor, has been associated with diverse biological functions in various organs and tissues [14]. EPO is already widely used clinically, so its use in novel clinical applications seems somewhat more practical than the use of growth factors, PRP, or ASCs. Several reports have focused the role of EPO in angiogenesis and wound healing, with promising results [15]. Recently, its possible positive role in lipofilling was also investigated in an in vitro model [16]. Considering its proangiogenic and antiapoptotic effects, we hypothesized that local delivery of EPO might enhance the survival of fat grafts in a nude mouse model.

\section{METHODS}

\section{Study design}

Our experiment consisted of 2 parts. In the first experiment, we performed daily local injections of EPO at the graft site immediately before fat grafting and for 4 consecutive days afterwards. In the second experiment, we inserted an osmotic pump at the graft site for continuous delivery of EPO to the grafted fat, for a longer period of time with a larger dose. All aspects of the protocol were approved by the Institutional Animal Care and Use Committee of Asan Institute for Life Sciences (2009-13-063). Additionally, all institutional and national guidelines for the care and use of laboratory animals were followed.

\section{Fat harvesting and processing}

Fat was obtained from the mid-abdomen of 2 healthy anonymized donors (44 and 46 years in age) who underwent elective abdominoplasty. Informed consent was obtained from each donor using an Institutional Review Board-approved protocol. Harvested fat was centrifuged at 3,000 rpm for 3 minutes, and the middle centrifugation layer was transferred to a 1-mL Luer-Lok syringe. The weight of each aliquot was calculated by subtracting the weight of an empty syringe from the total weight of the syringe containing fat.

\section{Animal model}

Thirty-six week-old CD-1 male nude mice (weight, 20-25 g) were used in this study. The animals were kept under a constant laminar flow of air and allowed to feed ad libitum on standard laboratory chow and water. For transplantation, the animal weight was mea- sured and $1 \mathrm{~mL}$ of fat was then injected into the subdermal scalp, using a sharp 16 gauge needles and spreading the fat through as large of an area as possible.

\section{Part 1. Local injection of EPO}

Eighteen mice were randomly divided into 2 experimental groups. Group 1E mice $(n=9)$ were injected subcutaneously once daily with EPO (Epokine ${ }^{\circledR}$ prefilled injection; CJ Pharmaceuticals, Seoul, Korea) at a dose of 5,000 IU/kg for 4 days around the graft site (100 IU daily for a $20 \mathrm{~g}$ mouse, about $10 \mu \mathrm{L}$ in volume) following fat injection. Group 1S mice $(n=9)$ were administered an equal volume of sterile normal saline based on body weight. The first injection was performed 1 hour before fat transplantation at the site of the presumed graft bed for mice in both groups.

\section{Part 2. Delivery of EPO via osmotic pump systems}

Eighteen animals were randomly divided into 3 subgroups: notreatment control (group 2C, $\mathrm{n}=6$ ), EPO delivered via an osmotic pump (ALZET ${ }^{\circledR}$ Model No. 1007D; ALZET Osmotic Pumps, Cupertino, CA, USA; group $2 \mathrm{E}, \mathrm{n}=6$ ), or sterile normal saline delivered via the same type of osmotic pump (group $2 S, n=6$ ). An osmotic pump containing 1,000 IU of EPO dissolved in $100 \mu \mathrm{L}$ of normal saline was connected to a polyethylene catheter (inner diameter, $0.76 \mathrm{~mm}$ ) and inserted in the subdermal lower back of the animal 1 hour before fat transplantation under anesthesia using 30 $\mathrm{mg} / \mathrm{kg}$ of Zoletil (Zoletil ${ }^{\mathbb{5} 50}$; Virbac Inc., Carros, France) administered intraperitoneally. The end of the catheter was placed at the recipient site and fixed with a fast-absorbing suture (Fig. 1). This osmotic pump system allowed continuous delivery of EPO at a rate

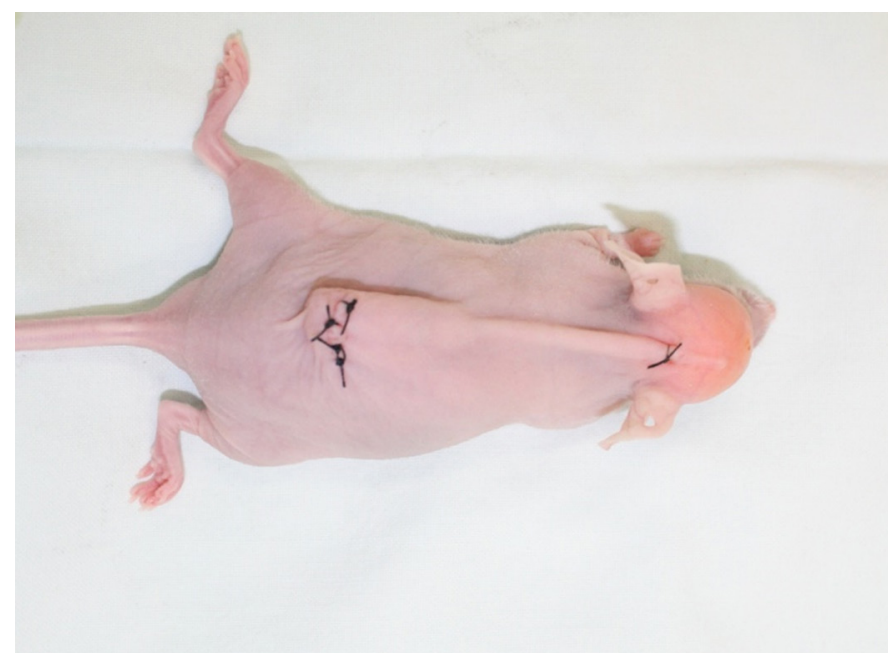

Fig. 1. Osmotic pump inserted into the grafted fat. The osmotic pump was connected to a polyethylene catheter and inserted in the subdermal lower back of the nude mouse. This osmotic pump system allowed continuous delivery of erythropoietin with a rate of $1 \mu \mathrm{L}(10$ IU of erythropoietin) per hour. 
of $1 \mu \mathrm{L}$ (10 IU of EPO) per hour (240 IU daily, for a total of 1,000 IU). The pump was surgically removed after 1 week through the back incision. A group without an osmotic pump served as the notreatment control group (group $2 \mathrm{C}, \mathrm{n}=6$ ).

\section{Data collection}

All animals survived to the end of this study and were euthanized after 16 weeks following the study protocol. The surviving fat from the original graft was dissected, weighed, and reported as the percent weight (\% weight) remaining from the initial injection weight. In cases where a macrocyst formed that contained necrotic material, the cyst portion, including the capsule, was excluded from the final weight measurement.

For the histological examinations, the central portion of dissected fat tissue was fixed in $4 \%$ formaldehyde, embedded in paraffin, and processed for hematoxylin and eosin staining. Five randomly selected fields from each slide were viewed by an independent observer to assess cellular integrity (the presence of nucleated fat cells), microvessel formation, cystic degeneration, fibrotic cicatrization, infiltration of inflammatory cells, and the presence of giant cells. Each parameter was graded on a semiquantitative scale $(0-5)$ as follows: 0 , absence; 1 , minimal; 2 , minimal to moderate; 3 , moderate; 4 , moderate to extensive; 5 , extensive presence.

\section{Statistical analysis}

Statistical analyses were conducted using SPSS version 20.0 (IBM Corp., Armonk, NY, USA). Repeated measures 1-way analysis of variance and the Mann-Whitney test were used to compare the percent weight and histological parameters. P-values of less than 0.05 were considered to indicate statistical significance in general.

Table 1. Comparison of graft survival and histomorphometric analysis results between group $1 \mathrm{E}$ and group $1 \mathrm{~S}$

\begin{tabular}{|c|c|c|c|}
\hline & 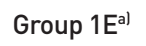 & Group $1 S^{b l}$ & P-value \\
\hline Surviving weight (\%) & 69.2 & 59.2 & 0.21 \\
\hline Cellular integrity & 4.44 & 4.11 & 0.40 \\
\hline Microvessel formation & 3.78 & 2.67 & $0.03^{*}$ \\
\hline Cystic degeneration & 0.56 & 1.78 & $0.04^{*}$ \\
\hline Fibrosis & 1.56 & 2.00 & 0.17 \\
\hline Cell infiltration & 1.67 & 1.78 & 0.72 \\
\hline Giant cells & 0.56 & 0.67 & 0.60 \\
\hline
\end{tabular}

Group 1E showed an increased tendency for graft weight retention, with significantly increased microvessel formation and less cystic degeneration than group 1S. The level of cellular infiltration and giant cell formation was comparable between the 2 groups.

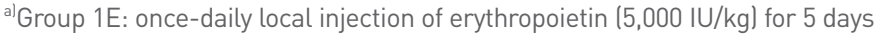
around the fat graft.

${ }^{b}$ Group 1S: once-daily local injection of normal saline $(500 \mu \mathrm{L} / \mathrm{kg})$ for 5 days around the fat graft.

*Significant difference $(P<0.05)$.
For intergroup comparisons, P-values $<0.01667$ (0.05/3) were considered to indicate statistical significance, following application of the post hoc Bonferroni correction.

\section{RESULTS}

\section{Part 1. Local injection of EPO}

The average percent weight of surviving fat was $69.2 \%$ in the EPOinjected group (1E) and 59.2\% in the saline-injected group (1S). Although fat graft survival was higher in the EPO-injected group, this tendency failed to reach statistical significance $(\mathrm{P}=0.21)$. All histological parameters were more favorable in the EPO-injected group (Table 1). Statistically significant differences were observed in microvessel formation and cystic degeneration (Fig. 2 and 3).

\section{Part 2. Delivery of EPO via an osmotic pump system}

The average percent weight of surviving fat was $40.7 \%, 7.7 \%$, and $66.7 \%$ in groups $2 \mathrm{E}, 2 \mathrm{~S}$, and $2 \mathrm{C}$, respectively. When each pair of groups was compared, group $2 \mathrm{E}$ showed a significantly higher survival rate than group $2 \mathrm{~S}(\mathrm{P}<0.001)$. However, both group $2 \mathrm{E}$ and $2 \mathrm{~S}$ group showed significantly poorer survival than the control group (group 2C; $\mathrm{P}<0.001, \mathrm{P}<0.0001$ ).

On histological evaluation, group $2 \mathrm{E}$ showed better overall results than group $2 \mathrm{~S}$, especially in terms of increased microvessel formation and fewer signs of inflammation. However, both osmotic pump groups (2E and $2 \mathrm{~S}$ ) showed a tendency for greater fibrosis and cellular infiltration than the control group (2C) (Table 2). Of note, group $2 \mathrm{~S}$ consistently showed a notable level of fibrosis with macrocystic degeneration in the histomorphometric analysis (Fig. 4).

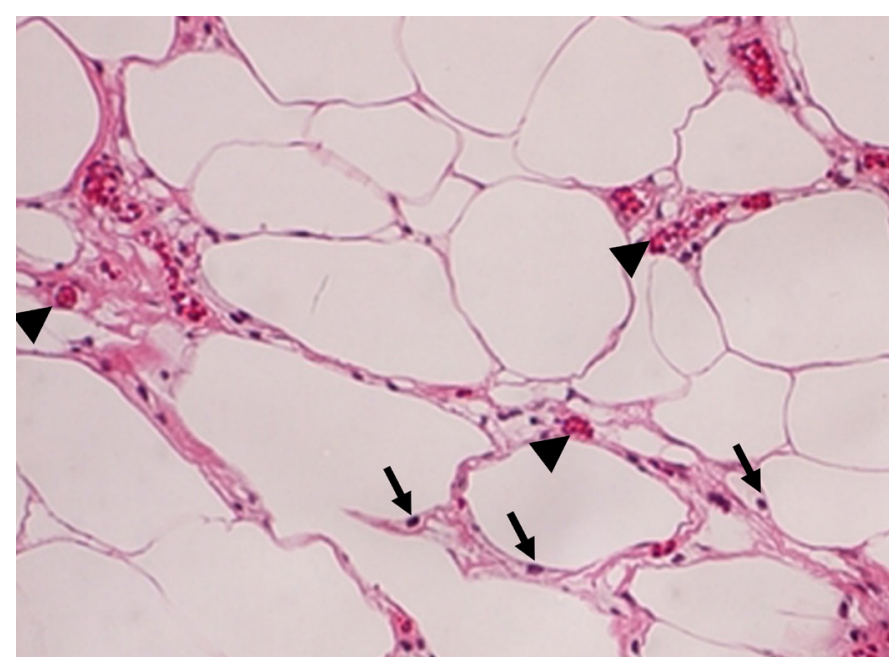

Fig. 2. Harvested fat from a mouse in group 1E. Surviving fat larrows, viable adipocyte nucleus) with little degeneration and microvessel formation (arrowheads) were observed (hematoxylin and eosin stain $[H \& E], \times 100)$. 


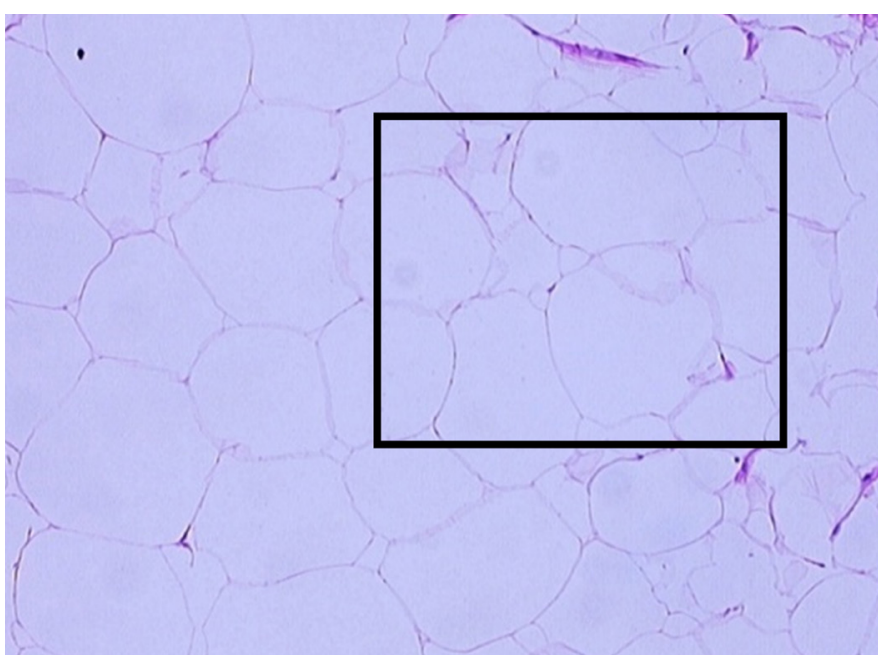

Fig. 3. Harvested fat from a mouse in group 1S, showing microcystic degeneration. Less microvessel formation, with scattered fibrosis and cystic degeneration (box, 'shadowy' outlines without nucleus), was observed (hematoxylin and eosin stain [H\&E], x100).

Table 2. Comparison of graft survival and histomorphometric analysis results in groups $2 \mathrm{E}, 2 \mathrm{~S}$, and $2 \mathrm{C}$

\begin{tabular}{|c|c|c|c|c|c|}
\hline & \multirow{2}{*}{$\begin{array}{l}\text { Group } \\
2 \mathrm{E}^{\mathrm{al}}\end{array}$} & \multirow{2}{*}{$\begin{array}{c}\text { Group } \\
2 S^{b /}\end{array}$} & \multirow{2}{*}{$\begin{array}{l}\text { Group } \\
2 C^{c l}\end{array}$} & \multicolumn{2}{|c|}{$\mathrm{P}$-value } \\
\hline & & & & 2E vs. $2 \mathrm{C}$ & $2 \mathrm{E}$ vs. $2 \mathrm{~S}$ \\
\hline Surviving weight (\%) & 40.7 & 7.7 & 66.7 & $<0.001$ & $<0.001$ \\
\hline Cellular integrity & 3.40 & 1.00 & 3.80 & 0.43 & $<0.001 *$ \\
\hline Microvessel formation & 3.40 & 2.00 & 3.17 & 0.64 & 0.15 \\
\hline Cystic degeneration & 1.80 & 3.25 & 1.17 & 0.12 & $0.002 *$ \\
\hline Fibrosis & 0.40 & 2.50 & 0.33 & 0.91 & $0.003^{*}$ \\
\hline Cell infiltration & 1.60 & 3.75 & 1.33 & 0.55 & $<0.001 *$ \\
\hline Giant cells & 0.83 & 1.83 & 0.33 & 0.07 & $0.009 *$ \\
\hline
\end{tabular}

Both pump groups showed significantly less graft weight retention than the no-treatment control group. The erythropoietin (EPO)-treated animals showed a greater tendency for microvessel formation, but less weight retention and cellular integrity than group 2 C. There was a consistent tendency for the EPOtreated animals to show worse histological features than group $2 \mathrm{C}$, although this trend did not reach statistical significance. The saline pump inserted animals showed significantly greater degeneration and inflammation/cicatrization than both other groups.

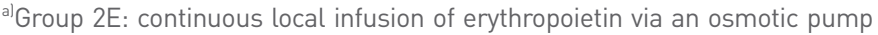
(10 IU per hour).

${ }^{b)}$ Group 2S: continuous local infusion of normal saline via an osmotic pump (1 $\mu \mathrm{L}$ per hour).

${ }^{c}$ Group 2C: no treatment (no osmotic pump).

*Significant difference $(P<0.05 / 3)$.

\section{DISCUSSION}

The main disadvantage of free fat injection is its unpredictable absorption rate. Ischemia that occurs prior to revascularization of injected fat causes adipocyte degeneration, leading to macrophage recruitment and subsequent phagocytosis of cellular debris. Vari-

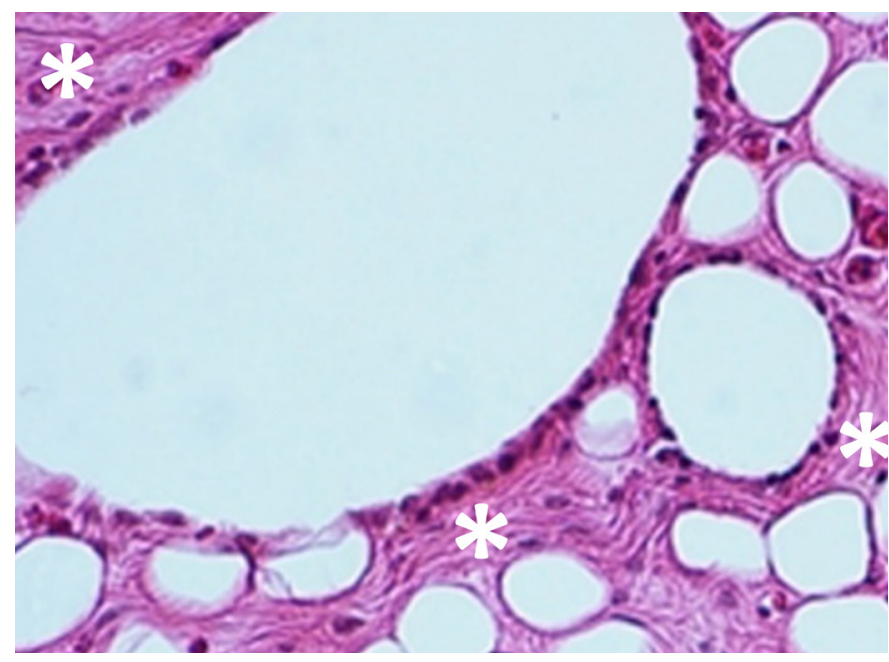

Fig. 4. Harvested fat from a mouse in group 2S. The large adipose nodule represents macrocystic degeneration. Oil cysts were scattered in areas of fibrotic cicatrization lasterisks, examples of severe fibrosis) which displaced healthy fat tissue and extracellular matrix (hematoxylin and eosin stain [H\&E], ×100).

ous proangiogenic factors and endothelial progenitor cells have been utilized in efforts to increase graft survival rates by enhancing the blood supply to the recipient area [8-10,17-20].

Although EPO is primarily known as a hemangiogenic factor, it plays a number of diverse biological roles in various organs and tissues, such as enhancing progenitor cell recruitment and inhibiting apoptosis. Animal studies have shown that EPO can protect against ischemia and reperfusion injury in various organs, including the heart, kidney, intestine, and liver [21-24]. EPO also promotes beneficial regeneration in neuronal tissue by enhancing progenitor cell recruitment and revascularization in injured regions $[25,26]$. This multifunctional factor has also been reported to enhance revascularization in an ischemic rat random flap and to increase survival in another rat random flap model $[27,28]$.

A recent in vitro study reported that EPO affected the inflammatory response by reducing the number of macrophages [16]. It also has anti-inflammatory and antioxidant properties, similarly to dimethyl sulfoxide, which showed beneficial effects on the survival and volume preservation of fat grafts $[29,30]$.

Hamed et al. [31] reported beneficial effects of pre- and subsequent treatment of EPO on the survival of grafted fat, and they claimed that the effect was even more potent than that of vascular endothelial growth factor. EPO treatment also increased the expression of angiogenic factors and microvascular density, and reduced inflammation and apoptosis [31]. These results are associated with both local and systemic effects, since subcutaneous injection of EPO in hemodialysis patients is known to be associated with more favorable clinical outcomes than intravenous treatment [32]. Based on these prior reports, we first tried to enhance the 
survival of fat grafts using local subcutaneous injections of highdose EPO (5,000 IU/kg per treatment) at the graft site, with a shorter treatment period (5 perioperative days vs. 18 days) in our first experiment. A trend towards a better histomorphometric profile was observed, as indicated by significantly increased microvessel formation and less cystic degeneration, which led to a tendency towards a higher survival rate than the saline-injected group, but the difference in fat graft survival was not significant.

However, in regenerative medicine research using animal models, continuous regional infusion of EPO using an osmotic pump was associated with enhanced functional recovery after global cerebral ischemia and protective neurogenesis in a model of Parkinson disease [33,34]. These protective effects have been proposed to be associated with several mechanisms, including the prevention of apoptosis and the stimulation of trophic factors. As the results from the first experiment showed possible benefits of EPO for increased fat survival, we hypothesized that the beneficial effect of continuous regional infusion of $\mathrm{EPO}$ on the neuronal system might also occur in the grafted fat tissue.

However, our set-up with an osmotic pump produced completely opposite results, as EPO delivery via an osmotic pump caused a significant reduction in the surviving graft weight compared to the no-treatment control group. The animals that received saline via an osmotic pump showed an even more dramatic reduction in fat graft survival.

Upon histological evaluation, the animals that were treated with either EPO or normal saline via the pump showed a marked increase in inflammatory cell infiltration and fibrosis. The addition of EPO seemed to have exerted a beneficial effect via its potent anti-inflammatory effect, as reflected by improved graft survival and a more favorable histological profile than the saline group. However, an exaggerated inflammatory response caused by local stimulation by the catheter itself, as well as an enhanced systemic inflammatory reaction, led to increased macrophage accumulation and subsequent destruction of fat cells. Similar results have been reported after the administration of interleukin-8, a proinflammatory mediator, which led to an improved histopathologic profile without an increase in the survival rate [9]. Haifei et al. [35] observed that the foreign body reaction, as demonstrated by giant cell formation, was the most extensive in the thickest scaffold and that vessel formation was the greatest in the thinnest scaffold, with the assumption that an enhanced cytokine response would have played a role in the degradation process, underlining the implications of inflammation and foreign body reaction in graft survival.

According to Kato et al. [36], initially 'dead' adipocytes are replaced by next-generation adipocytes in favorable microenvironments. An excessive inflammatory reaction seems to expand the necrotizing zone and to shrink the regenerating zone because the acute inflammatory response involving cellular influx leads to cell and tissue damage and subsequent resorption by macrophages.
When lipid replacement with scar tissue predominates, fibrosis occurs, further hindering adipose stem cell differentiation [37].

Although fat graft survival depends on revascularization, resorption of the graft is not solely dependent on the amount of blood supply. An exaggerated inflammatory response before complete revascularization may lead to significant resorption of the grafted fat and cicatrization, as in the current study. Similar results were recently published by Cai et al. [37] who reported that low-dose granulocyte colony-stimulating factor improved fat graft retention by mobilizing stem cells and inducing angiogenesis, whereas high doses inhibited adipogenesis, with prolonged inflammation and severe fibrosis. In short, fat graft survival requires an appropriate inflammatory response at the early stage to initiate precursor cell proliferation and angiogenesis, followed by quick remission to prevent excessive phagocytosis and fibrosis.

Although it failed to reach statistical significance, we showed some possible benefits of EPO, and more importantly, the significantly detrimental effect of excessive or prolonged inflammation on the survival of fat grafts. Future studies on the effects of beneficial agents or growth factors should employ a benign delivery method such as direct injection, mixing with the graft, or the use of microspheres, as reported by previous studies [38,39]. In clinical practice, the principle of minimizing the inflammatory reaction should be considered when fat grafting is performed with other procedures, especially when an alloplastic material or prosthesis is being concurrently implanted.

\section{REFERENCES}

1. Marino G, Moraci M, Armenia E, et al. Therapy with autologous adipose-derived regenerative cells for the care of chronic ulcer of lower limbs in patients with peripheral arterial disease. J Surg Res 2013;185: 36-44.

2. Khouri RK, Smit JM, Cardoso E, et al. Percutaneous aponeurotomy and lipofilling: a regenerative alternative to flap reconstruction? Plast Reconstr Surg 2013;132:1280-90.

3. Fisher C, Grahovac TL, Schafer ME, et al. Comparison of harvest and processing techniques for fat grafting and adipose stem cell isolation. Plast Reconstr Surg 2013;132:351-61.

4. Gir P, Brown SA, Oni G, et al. Fat grafting: evidence-based review on autologous fat harvesting, processing, reinjection, and storage. Plast Reconstr Surg 2012;130:249-58.

5. Condé-Green A, de Amorim NF, Pitanguy I. Influence of decantation, washing and centrifugation on adipocyte and mesenchymal stem cell content of aspirated adipose tissue: a comparative study. J Plast Reconstr Aesthet Surg 2010;63:1375-81.

6. Yamaguchi M, Matsumoto F, Bujo H, et al. Revascularization determines volume retention and gene expression by fat grafts in mice. Exp Biol Med (Maywood) 2005;230:742-8.

7. Park B, Kong JS, Kang S, et al. The effect of epidermal growth factor 
on autogenous fat graft. Aesthetic Plast Surg 2011;35:738-44.

8. Chung CW, Marra KG, Li H, et al. VEGF microsphere technology to enhance vascularization in fat grafting. Ann Plast Surg 2012;69:213-9.

9. Shoshani O, Livne E, Armoni M, et al. The effect of interleukin-8 on the viability of injected adipose tissue in nude mice. Plast Reconstr Surg 2005;115:853-9.

10. Cervelli V, Scioli MG, Gentile P, et al. Platelet-rich plasma greatly potentiates insulin-induced adipogenic differentiation of human adiposederived stem cells through a serine/threonine kinase Akt-dependent mechanism and promotes clinical fat graft maintenance. Stem Cells Transl Med 2012;1:206-20.

11. Serra-Mestre JM, Serra-Renom JM, Martinez L, et al. Platelet-rich plasma mixed-fat grafting: a reasonable prosurvival strategy for fat grafts? Aesthetic Plast Surg 2014;38:1041-9.

12. Piccinno MS, Veronesi E, Loschi $\mathrm{P}$, et al. Adipose stromal/stem cells assist fat transplantation reducing necrosis and increasing graft performance. Apoptosis 2013;18:1274-89.

13. Begic A, Isfoss BL, Lønnerød LK, et al. Survival and inflammatory response in adipose-derived mesenchymal stem cell-enriched mouse fat grafts. Plast Reconstr Surg Glob Open 2016;4:e1110.

14. Arcasoy MO. The non-haematopoietic biological effects of erythropoietin. Br J Haematol 2008;141:14-31.

15. Sayan H, Ozacmak VH, Guven A, et al. Erythropoietin stimulates wound healing and angiogenesis in mice. J Invest Surg 2006;19:163-73.

16. Sabbatini M, Moalem L, Bosetti M, et al. Effects of erythropoietin on adipose tissue: a possible strategy in refilling. Plast Reconstr Surg Glob Open 2015;3:e338.

17. Baran CN, Celebioğlu S, Sensöz O, et al. The behavior of fat grafts in recipient areas with enhanced vascularity. Plast Reconstr Surg 2002; 109:1646-51; 52.

18. Hamed S, Ben-Nun O, Egozi D, et al. Treating fat grafts with human endothelial progenitor cells promotes their vascularization and improves their survival in diabetes mellitus. Plast Reconstr Surg 2012;130:801-11.

19. Eppley BL, Sidner RA, Platis JM, et al. Bioactivation of free-fat transfers: a potential new approach to improving graft survival. Plast Reconstr Surg 1992;90:1022-30.

20. Yi C, Pan Y, Zhen Y, et al. Enhancement of viability of fat grafts in nude mice by endothelial progenitor cells. Dermatol Surg 2006;32:1437-43.

21. Riksen NP, Hausenloy DJ, Yellon DM. Erythropoietin: ready for primetime cardioprotection. Trends Pharmacol Sci 2008;29:258-67.

22. Ma YS, Zhou J, Liu H, et al. Protection effect of recombiant human erythropoietin preconditioning against intrauterine hypoxic-ischemic brain injury and its influence on expression of caspase-3 protein in brain tissue. Sichuan Da Xue Xue Bao Yi Xue Ban 2013;44:397-401.

23. Schmeding M, Boas-Knoop S, Lippert S, et al. Erythropoietin promotes hepatic regeneration after extended liver resection in rats. J Gastroenterol Hepatol 2008;23:1125-31.

24. Mori S, Sawada T, Okada T, et al. Erythropoietin and its derivative protect the intestine from severe ischemia/reperfusion injury in the rat. Surgery 2008;143:556-65.
25. Pankratova S, Gu B, Kiryushko D, et al. A new agonist of the erythropoietin receptor, Epobis, induces neurite outgrowth and promotes neuronal survival. J Neurochem 2012;121:915-23.

26. Toth C, Martinez JA, Liu WQ, et al. Local erythropoietin signaling enhances regeneration in peripheral axons. Neuroscience 2008;154:76783.

27. Buemi M, Vaccaro M, Sturiale A, et al. Recombinant human erythropoietin influences revascularization and healing in a rat model of random ischaemic flaps. Acta Derm Venereol 2002;82:411-7.

28. Saray A, Ozakpinar R, Koc C, et al. Effect of chronic and short-term erythropoietin treatment on random flap survival in rats: an experimental study. Laryngoscope 2003;113:85-9.

29. Sari E, Bakar B, Sarkarati B, et al. Effectiveness of dimethylsulfoxide on the survival and volume preservation of autologous fat graft tissue: a preliminary study. Aesthet Surg J 2016;36:Np58-67.

30. Bailey DM, Lundby C, Berg RM, et al. On the antioxidant properties of erythropoietin and its association with the oxidative-nitrosative stress response to hypoxia in humans. Acta Physiol (Oxf) 2014;212:175-87.

31. Hamed S, Egozi D, Kruchevsky D, et al. Erythropoietin improves the survival of fat tissue after its transplantation in nude mice. PLoS One 2010;5:e13986.

32. Wright DG, Wright EC, Narva AS, et al. Association of erythropoietin dose and route of administration with clinical outcomes for patients on hemodialysis in the United States. Clin J Am Soc Nephrol 2015;10: 1822-30.

33. Undén J, Sjölund C, Länsberg JK, et al. Post-ischemic continuous infusion of erythropoeitin enhances recovery of lost memory function after global cerebral ischemia in the rat. BMC Neurosci 2013;14:27.

34. Kadota T, Shingo T, Yasuhara T, et al. Continuous intraventricular infusion of erythropoietin exerts neuroprotective/rescue effects upon Parkinson's disease model of rats with enhanced neurogenesis. Brain Res 2009;1254:120-7.

35. Haifei S, Xingang W, Shoucheng W, et al. The effect of collagen-chitosan porous scaffold thickness on dermal regeneration in a one-stage grafting procedure. J Mech Behav Biomed Mater 2014;29:114-25.

36. Kato H, Mineda K, Eto H, et al. Degeneration, regeneration, and cicatrization after fat grafting: dynamic total tissue remodeling during the first 3 months. Plast Reconstr Surg 2014;133:303e-13e.

37. Cai J, Li B, Liu K, et al. Low-dose G-CSF improves fat graft retention by mobilizing endogenous stem cells and inducing angiogenesis, whereas high-dose G-CSF inhibits adipogenesis with prolonged inflammation and severe fibrosis. Biochem Biophys Res Commun 2017;491:662-7.

38. Yuksel E, Weinfeld AB, Cleek R, et al. Increased free fat-graft survival with the long-term, local delivery of insulin, insulin-like growth factor-I, and basic fibroblast growth factor by PLGA/PEG microspheres. Plast Reconstr Surg 2000;105:1712-20.

39. Chang L, Wang J, Zheng D, et al. Improvement of the survival of autologous free-fat transplants in rats using vascular endothelial growth factor 165-transfected bone mesenchymal stem cells. Ann Plast Surg 2014;72:355-62. 